\title{
When is a Copula Constant? A Test for Changing Relationships
}

Fabio Busetti and Andrew Harvey

September 2008

CWPE 08341 


\title{
When is a copula constant? A test for changing relationships
}

\author{
Fabio Busetti and Andrew Harvey \\ Bank of Italy and Faculty of Economics, Cambridge*
}

August 4, 2008

\begin{abstract}
A copula defines the probability that observations from two time series lie below given quantiles. It is proposed that stationarity tests constructed from indicator variables be used to test against the hypothesis that the copula is changing over time. Tests associated with different quantiles may point to changes in different parts of the copula, with the lower quantiles being of particular interest in financial applications concerned with risk. Tests located at the median provide an overall test of a changing relationship. The properties of vari-
\end{abstract}


ous tests are compared and it is shown that they are still effective if pre-filtering is carried out to correct for changing volatility or, more generally, changing quantiles. Applying the tests to daily stock return indices in Korea and Thailand over the period 1995-9 indicates that the relationship between them is not constant over time.

KEYWORDS: Concordance; quantile; rank correlation; stationarity test; tail dependence.

* ACH34@ECON.CAM.AC.UK

\section{Introduction}

Understanding and measuring the relationship between movements in different assets plays a key role in designing a portfolio. The multivariate normal distribution may not be suitable for this task for two reasons: asset returns are not normally distributed and their comovements are not adequately captured by correlation coefficients.

A more general description of dependence is given by the proportion of cases in which the observation on one series is below (above) a given quantile, given that the observation on the other series is below (above) a given quantile. Looking at different quantiles allows us to focus on different aspects of 
the relationship. The underlying probabilities are given by the copula, which is a joint distribution function with uniform marginals.

The copula may change over time. Evidence that this might happen is provided by Van Der Goorbergha, Genest and Werker (2005) and Patton (2006). Rodriguez (2007), in his study of Asian and Latin American stock indices, finds evidence of changing dependence during periods of turmoil and concludes as follows. 'Changes in tail dependence should be taken into account in the design of any sound asset allocation strategy. Failing to do so can be expensive, as recent theoretical literature has demonstrated. Moreover, it is important to note that these changes are not necessarily captured by correlation shifts.' Das and Upal (2004) highlight the costs of ignoring regime shifts for asset allocation. Cherubini et al (2004, p 73-4) discuss the relevance for the value at risk $(\mathrm{VaR})$ of a portfolio.

The aim of this paper is to develop tests for changes in different parts of the copula, as well as overall tests for changing dependence. These tests do not require a model for the copula and they can be regarded as an extension of the stationarity tests for time-varying quantiles proposed in Busetti and Harvey (2007). The test statistics are constructed from time series of indicator variables and their asymptotic distributions under the null hypothesis 
come from the family of Cramér-von Mises distributions. Most of the tests in the literature, for example in studies of contagion, have been concerned with tests of changes at known breakpoints. The tests proposed here have power against breaks at unknown points as well as against gradual, but persistent, changes.

Section 2 of the paper reviews the tests proposed in Busetti and Harvey (2007) for individual series. The tests proposed for a changing copula are described in section 3. Section 4 reports Monte Carlo experiments. Section 5 notes that the tests will be affected if the marginal distributions change over time. A set of Monte Carlo experiments investigate the performance of the tests when pre-filtering is carried out to allow for changing volatility in the individual series. More generally we discuss how to correct for time-variation in the quantiles of the individual series. However, the preferred overall test for changing dependence is based on medians and if these are constant the test is not affected by movements in other parts of the distribution. The application is in section 6 , while section 7 concludes. 


\section{Quantiles and Quantics}

Let $\xi(\tau)$ denote the $\tau-$ th quantile. The probability that an observation is less than $\xi(\tau)$ is $\tau$, where $0<\tau<1$. Given a set of $T$ observations, $y_{t}, t=1, . ., T$, the sample quantile, $\widetilde{\xi}(\tau)$, can be obtained by sorting the observations in ascending order. Since several observations may coincide with a sample quantile, a general definition of a sample $\tau$-quantile is a point such that the number of observations smaller is no more than $[T \tau]$ while the number greater is no more than $[T(1-\tau)]$.

The residuals associated with a quantile may be coded as indicators. The $\tau$-quantile indicator is

$$
I Q\left(y_{t}-\xi(\tau)\right)=\left\{\begin{array}{cc}
\tau-1, & \text { if } y_{t}<\xi(\tau) \\
\tau, & \text { if } y_{t}>\xi(\tau)
\end{array}, \quad t=1, \ldots, T\right.
$$

Note that $I Q(0)$ is not determined but we will constrain it to lie in the range $[\tau-1, \tau]$

A test of the null hypothesis that a quantile is constant may be based on the sample $\tau$-quantile indicators, or $\tau$-quantics, that is $I Q\left(y_{t}-\widetilde{\xi}(\tau)\right), t=$ $1, \ldots, T$. If the alternative hypothesis is that the $\tau$-quantile follows a random 
walk, a modified version of the basic stationarity test of Nyblom and Mäkeläinen (1983) is appropriate. The test statistic of Nyblom and Mäkeläinen uses residuals from a sample mean and its asymptotic distribution is a Cramérvon Mises $(C v M)$ distribution; the $1 \%, 5 \%$ and $10 \%$ critical values are 0.743 , 0.461 and 0.347 respectively. Nyblom and Harvey (2001) show that the test has high power against an integrated random walk, which when fitted yields a curve close to a cubic spline, while Harvey and Streibel (1998) show that it also has a locally best invariant interpretation as a test of constancy against a highly persistent stationary first-order autoregressive process. Note also that, as shown in Nyblom (1989), the test has power against a break, or breaks, in an otherwise stationary time series.

Busetti and Harvey (2007) show that under the null hypothesis that the observations are IID and $\xi(\tau)$ is the unique population $\tau$-quantile and $y$ has a continuous positive density in the neighborhood of $\xi(\tau)$, the asymptotic distribution of the quantic-based stationarity test statistic

$$
\eta_{\tau}(Q)=\frac{1}{T^{2} \tau(1-\tau)} \sum_{t=1}^{T}\left(\sum_{i=1}^{t} I Q\left(y_{i}-\widetilde{\xi}(\tau)\right)\right)^{2}
$$

is the $C v M$ distribution. The proof extends the one in De Jong, Amsler and 
Schmidt (2007) for $\tau=0.5$. As in that paper, allowance can be made for serial correlation by replacing $\tau(1-\tau)$ by a nonparametric estimator of the spectrum at zero frequency.

A joint test to see if a group of $N$ quantiles show evidence of changing over time can be based on a generalization of (2). Under the null hypothesis of IID observations, the limiting distribution of this multivariate test statistic is Cramér-von Mises with $N$ degrees of freedom.

Linton and Whang (2007) suggest that correlograms be constructed from quantics. They call these quantilograms and suggest that Box-Ljung tests be carried out for serial correlation.

\section{Bivariate series}

Consider a bivariate series, $y_{1 t}$ and $y_{2 t}, t=1, \ldots, T$. By converting to ranks we can obtain the sample quantiles and the empirical copula. The empirical copula yields the proportion of cases in which both observations in a pair are

less than, or equal to, particular quantiles, $\widetilde{\xi}\left(\tau_{1}\right)$ and $\widetilde{\xi}\left(\tau_{2}\right)$. This proportion will be denoted as $C_{T}\left(\tau_{1}, \tau_{2}\right)$.

The tests in Busetti and Harvey (2007) were designed to detect move- 
ments in the quantiles of the distributions of univariate series. If there are two series and their marginal distributions are constant, we can move on to address the question of whether their copula is changing over time. As with univariate series, the tests are based on indicators, but we now have to consider combinations of quantiles from the two series. To simplify matters, we will set $\tau_{1}=\tau_{2}=\tau, 0<\tau<1$, and explore the possibility of movements in $C(\tau, \tau)$, the probability that both observations lie below their respective $\tau$-quantiles. However, since there are four quadrants associated with a given $\tau$, some attention needs to be paid to the other three. The probability that both observations lie above their respective $\tau$-quantiles is known as the survival function, and denoted $\bar{C}(\tau, \tau)$. It is equal to $1-2 \tau+C(\tau, \tau)$; see Embrechts et al (2003) or Cherubini et al (2004, p75). The probabilities of being in the other two quadrants are the same, namely $\tau-C(\tau, \tau)$. Similar relationships hold for the corresponding sample proportions.

\subsection{Copulas and concordance}

The value of $C(\tau, \tau)$ indicates the strength of dependence at $\tau$. With perfect concordance, $C(\tau, \tau)=\tau$ and $\bar{C}(\tau, \tau)=1-\tau$, while with perfect discordance

both these probabilities are zero. For independent series, $C(\tau, \tau)=\tau^{2}$, while 
$\bar{C}(\tau, \tau)=(1-\tau)^{2}$, and the probabilities of being in one of the other quadrants are both $\tau(1-\tau)$. Summing $C(\tau, \tau)$ and $\bar{C}(\tau, \tau)$ gives a simple measure of what Kruskal (1958, p 818) calls quadrant association.

As an illustration, the Clayton copula is defined in terms of standard uniform variates, $u_{1}$ and $u_{2}$, as

$$
C\left(u_{1}, u_{2}\right)=\left(u_{1}^{-\theta}+u_{2}^{-\theta}-1\right)^{-1 / \theta}, \quad \theta \in[-1, \infty)
$$

For $\theta=1$ and a small $\tau, C(\tau, \tau) \simeq \tau / 2$. The Clayton copula is asymmetric in that the upper tail probability for $1-\tau$, that is the survival copula, $\bar{C}(1-\tau, 1-\tau)$, is not the same as $C(\tau, \tau)$. The relationship is

$$
\bar{C}(1-\tau, 1-\tau)=2 \tau-1+(1-\tau)\left(2-(1-\tau)^{\theta}\right)^{-1 / \theta}
$$

For $\theta=1, \bar{C}(1-\tau, 1-\tau)$ is .018 for $\tau=.1$, while for $\tau=.05$, it is .0048 . These probabilities are much smaller than those for the lower tail.

The coefficients of tail dependence are measures of pairwise dependence that depend on the copula; see McNeil et al (2005, p208). The coefficient of lower tail dependence is $\lim _{\tau \rightarrow 0} C(\tau, \tau) / \tau$, while the coefficient of upper tail dependence is $\lim _{\tau \rightarrow 1} \bar{C}(\tau, \tau) /(1-\tau)$. If two variables have a bivariate normal 
distribution, they are asymptotically independent in the tails as the coefficients of tail dependence are both zero. On the other hand, a t-copula does exhibit tail dependence. For $\theta>0$, the Clayton copula exhibits lower tail dependence, that is $\lim _{\tau \rightarrow 0} C(\tau, \tau) / \tau=2^{-1 / \theta}$, and as $\theta \rightarrow \infty$, this coefficient tends to one.

\subsection{Bivariate quantics}

We will define the bivariate $\tau$-quantic - or $\tau$-bi-quantic - as

$$
\begin{aligned}
\operatorname{BIQ}\left(y_{1 t}\right. & \left.\leq \widetilde{\xi}_{1}(\tau), y_{2 t} \leq \widetilde{\xi}_{2}(\tau)\right) \\
& =C_{T}(\tau, \tau)-I\left(y_{1 t} \leq \widetilde{\xi}_{1}(\tau), y_{2 t} \leq \widetilde{\xi}_{2}(\tau)\right), \quad t=1, \ldots, T
\end{aligned}
$$

where $I\left(y_{1 t} \leq \widetilde{\xi}_{1}(\tau), y_{2 t} \leq \widetilde{\xi}_{2}(\tau)\right)$ denotes $I\left(y_{1 t} \leq \widetilde{\xi}_{1}(\tau)\right) \cdot I\left(y_{2 t} \leq \widetilde{\xi}_{2}(\tau)\right.$ with $I($.$) being the indicator function. By construction B I Q\left(y_{1 t} \leq \widetilde{\xi}_{1}(\tau), y_{2 t} \leq\right.$ $\left.\widetilde{\xi}_{2}(\tau)\right)$ has a mean of zero, since $I\left(y_{1 t} \leq \widetilde{\xi}_{1}(\tau), y_{2 t} \leq \widetilde{\xi}_{2}(\tau)\right) / T=C_{T}(\tau, \tau)$, and a variance of $C_{T}(\tau, \tau)\left(1-C_{T}(\tau, \tau)\right)$. When the bivariate observations, $\left(y_{1 t}, y_{2 t}\right)$, are ${ }^{1}$ independently and identically distributed (IID), the corresponding bivariate $\tau$-quantile indicator, $B I Q\left(y_{1 t} \leq \xi_{1}(\tau), y_{2 t} \leq \xi_{2}(\tau)\right)$, has

\footnotetext{
${ }^{1}$ See the assumption in appendix A.
} 
a mean of zero and a variance of $C(\tau, \tau)(1-C(\tau, \tau))$. When there is no ambiguity we will abbreviate $\operatorname{BIQ}\left(y_{1 t} \leq \widetilde{\xi}_{1}(\tau), y_{2 t} \leq \widetilde{\xi}_{2}(\tau)\right.$ to $\operatorname{BIQ}(\tau)$.

Associated with $B I Q(\tau)$ are three complementary bivariate $\tau$-quantics. The first is

$$
\operatorname{BIQ}\left(y_{1 t}>\widetilde{\xi}_{1}(\tau), y_{2 t}>\widetilde{\xi}_{2}(\tau)\right)=\bar{C}_{T}(\tau, \tau)-I\left(y_{1 t}>\widetilde{\xi}_{1}(\tau), y_{2 t}>\widetilde{\xi}_{2}(\tau)\right)
$$

We call this the survival $\tau-$ quantic and use the shorthand notation $\overline{B I Q}(\tau)$. Remember that $\bar{C}_{T}(\tau, \tau)=1-2 \tau+C_{T}(\tau, \tau)$. The variance of $\overline{B I Q}(\tau)$ is $\bar{C}_{T}(\tau, \tau)\left(1-\bar{C}_{T}(\tau, \tau)\right)$. The other two complementary $\tau$-bi-quantics are

$$
B I Q\left(y_{1 t} \leq \widetilde{\xi}_{1}(\tau), y_{2 t}>\widetilde{\xi}_{2}(\tau)\right)=\bar{C}_{T}^{*}(\tau, \tau)-I\left(y_{1 t} \leq \widetilde{\xi}_{1}(\tau), y_{2 t}>\widetilde{\xi}_{2}(\tau)\right)
$$

and

$$
B I Q\left(y_{1 t}>\widetilde{\xi}_{1}(\tau), y_{2 t} \leq \widetilde{\xi}_{2}(\tau)\right)=\bar{C}_{T}^{*}(\tau, \tau)-I\left(y_{1 t}>\widetilde{\xi}_{1}(\tau), y_{2 t} \leq \widetilde{\xi}_{2}(\tau)\right)
$$

where $\bar{C}_{T}^{*}(\tau, \tau)=\tau-C_{T}(\tau, \tau)$.

Note the identities that relate $\tau$-bi-quantics to the $\tau$-quantics in the 
individual series:

$B I Q\left(y_{1 t} \leq \widetilde{\xi}_{1}(\tau), y_{2 t} \leq \widetilde{\xi}_{2}(\tau)\right)+B I Q\left(y_{1 t} \leq \widetilde{\xi}_{1}(\tau), y_{2 t}>\widetilde{\xi}_{2}(\tau)\right)=I Q\left(y_{1 t} \leq \widetilde{\xi}_{1}(\tau)\right)$

and

$B I Q\left(y_{1 t} \leq \widetilde{\xi}_{1}(\tau), y_{2 t} \leq \widetilde{\xi}_{2}(\tau)\right)+B I Q\left(y_{1 t}>\widetilde{\xi}_{1}(\tau), y_{2 t} \leq \widetilde{\xi}_{2}(\tau)\right)=I Q\left(y_{2 t} \leq \widetilde{\xi}_{2}(\tau)\right)$

Expressions for the correlations between the four bivariate $\tau$-quantile indicators can be obtained when the bivariate series is IID; see appendix A. For example, the correlation between $B I Q\left(y_{1 t} \leq \xi_{1}(\tau), y_{2 t} \leq \xi_{2}(\tau)\right)$ and $B I Q\left(y_{1 t}>\xi_{1}(\tau), y_{2 t}>\xi_{2}(\tau)\right)$ is

$$
\frac{-\sqrt{C(\tau, \tau)} \sqrt{1-2 \tau+C(\tau, \tau)}}{\sqrt{1-C(\tau, \tau)} \sqrt{2 \tau-C(\tau, \tau)}}
$$

If the original series are independent of each other, then $C(\tau, \tau)=\tau^{2}$ and (8) becomes $-\sqrt{\tau(1-\tau)} / \sqrt{(2-\tau)(1+\tau)}$. Thus for $\tau=0.5$ the correlation is $-1 / 3$. 


\subsection{Tests}

We now consider tests of the null hypothesis that $C(\tau, \tau)$ is constant. Stationarity test statistics can be formed from the bi-quantics in the same way as for the quantics. Thus, for a given $\tau$,

$$
\eta_{\tau}(B Q ; B B)=\frac{1}{T^{2} C_{T}(\tau, \tau)\left(1-C_{T}(\tau, \tau)\right)} \sum_{t=1}^{T}\left(\sum_{i=1}^{t} B I Q(\tau)\right)^{2}
$$

where $B B$ signifies that the indicator is unity when both observations are below their respective quantiles. The asymptotic distribution of this stationarity test statistic when the bivariate series is IID is Cramér von Mises. The proof is given in appendix B. A non-parametric correction for serial correlation may be made, as in De Jong et al (2007), by replacing $C_{T}(\tau, \tau)(1-$ $C_{T}(\tau, \tau)$ ) with a long-run variance estimator for $B I Q(\tau)$. The asymptotic distribution continues to be Cramér von Mises for bivariate observations that are jointly strictly stationary. A correction for serial correlation is not needed if the test is being used to detect any kind of time-variation, but permanent changes have more serious consequences. Since there are limits on the range of $C(\tau, \tau)$ the alternative hypothesis is best thought of as one in which $C(\tau, \tau)$ 
is slowly changing, or subject to breaks, rather than being a random walk.

Tests statistics for the other bi-quantics may be formed in a similar way. The test statistic constructed using the survival bi-quantics will be denoted $A A$ (for above), while the other two are $B A$ and $A B$. These individual quadrant tests might point to movements in some parts of the copula but not in others. For example it may be interesting to contrast lower and upper tail movements from $\eta_{\tau}(B Q ; B B)$ and $\eta_{1-\tau}(B Q ; A A)$.

A combined test can be constructed from any three bi-quantics, provided that $C_{T}(\tau, \tau)<\tau$. The statistic is

$$
\eta_{\tau}(B Q ; 3)=T^{-2} \sum_{t=1}^{T}\left[\sum_{i=1}^{t} \mathbf{B I Q}_{i}\right]^{\prime} \widetilde{\Omega}^{-1}\left[\sum_{i=1}^{t} \mathbf{B I Q}_{i}\right]
$$

where the elements of the $3 \times 1$ vector $\mathbf{B I Q}_{i}$ are chosen from (4), (5), (6) and (7), and $\widetilde{\Omega}$ is their sample covariance matrix. Under the null hypothesis of serially independent observations with a constant copula, the limiting distribution of $\eta_{\tau}(B Q ; 3)$ is Cramér-von Mises with three degrees of freedom. (The proof is straightforward given the proof for tests based on a single biquantic). The $1 \%, 5 \%$ and $10 \%$ critical values are $1.359,1.000$ and 0.841 respectively 
A second test is based on noting that, even if the copula changes, there is a symmetry between the 'off-diagonal' quadrants in that pairs of observations always fall in them with equal probability. Thus (6) and (7) could be added together to make a new series and a test statistic formed from two of the three series. Since the choice of series does not matter, the easiest way to proceed is to construct the test statistic, $\eta_{\tau}(B Q ; 2)$, from $B I Q(\tau)$ and $\overline{B I Q}(\tau)$, in a similar way to (10). Its limiting distribution under the null hypothesis is Cramér-von Mises with 2 degrees of freedom.

Finally we can add $B I Q(\tau)$ and $\overline{B I Q}(\tau)$ to give

$$
\begin{gathered}
B I Q(\tau)+\overline{B I Q}(\tau)= \\
1-2 \tau+2 C_{T}(\tau, \tau)-I\left(y_{1 t} \leq \widetilde{\xi}_{1}(\tau)\right) \cdot I\left(y_{2 t} \leq \widetilde{\xi}_{2}(\tau)\right)-I\left(y_{1 t}>\widetilde{\xi}_{1}(\tau)\right) \cdot I\left(y_{2 t}>\widetilde{\xi}_{2}(\tau)\right) .
\end{gathered}
$$

A test statistic formed from this series will be denoted $\eta_{\tau}(B Q+\overline{B Q})$. Since it is based on the sum of $C_{T}(\tau, \tau)$ and $\bar{C}_{T}(\tau, \tau)$, we will call it the quadrant association test. Its limiting distribution under the null hypothesis is Cramér-von Mises with one degree of freedom. 


\subsection{Single comprehensive tests of changing dependence}

If a single overall test for changing dependence is required, it may be based on the combined or quadrant association tests for $\tau=0.5$. The choice of $\tau=0.5$ seems eminently reasonable and is supported by the Monte Carlo simulations. We might compare the power of these tests with a stationarity test based on the series $\left(y_{1 t}-\bar{y}_{1}\right)\left(y_{2 t}-\bar{y}_{2}\right), t=1, \ldots, T$. We call this the changing correlation test. The limiting distribution of the changing correlation test statistic is Cramér-von Mises with one degree of freedom under the null hypothesis that $y_{1 t}$ and $y_{2 t}$ are serially independent with a constant, finite, positive definite, covariance matrix. The usual conditions on the existence of moments have to be carried over to $y_{1 t} y_{2 t}$. A non-parametric correction for serial correlation may be made, as in Kwiatkowski et al (1992), and the proof correspondingly extended to deal with a null hypothesis of joint stationarity. The performance of the changing correlation test statistic will depend on the whole joint distribution, not just the copula, and it may be quite sensitive to extreme observations.

While the tests at different $\tau$ may provide information on changes in different parts of the copula, a combination of them may be effective as an overall test. For example, a multivariate quadrant association test statistic 
may be constructed from $B I Q(\tau)+\overline{B I Q}(\tau)$ for $\tau=0.25,0.5$ and 0.75 . Another possibility is to combine $B I Q(0.5)+\overline{B I Q}(0.5)$ with $B I Q(0.25)$ and $\overline{B I Q}(0.75)$.

\subsection{Changing contrasts}

A joint test of changes in the upper and lower tails can be based on $B I Q(\tau)+$ $\overline{B I Q}(1-\tau)$. A test of changing asymmetry can be based on $B I Q(\tau)-$ $\overline{B I Q}(1-\tau)$. The limiting distribution of the both (stationarity) test statistics is Cramér-von Mises with one degree of freedom under the null hypothesis that the underlying probabilities do not change.

\subsection{Bi-quantilogram}

The pattern of serial correlation can be captured by computing the correlograms of the bivariate $\tau$-quantics. These might be called bi-quantilograms. They are not the same as the cross-quantilograms of Linton and Whang (2007), though these may also be useful. Box-Ljung Q-statistics may be formed from the bi-quantilograms and used as an alternative to stationarity tests for assessing change in the copula. 


\section{Monte Carlo}

We consider three cases of data generating processes, denoted by A,B,C; the first two are based on a time-varying Gaussian copula while the third is characterized by a Clayton copula, with lower tail dependence. In general the two degree of freedom test was dominated by the others and so we do not report the results for it. All tests are at the $5 \%$ significance level and are based on 20,000 replications. The program were written in Ox and made use of the routines in SSFpack; see Doornik (1999) and Koopman et al (1999).

The speed of convergence to the asymptotic distribution may be slow when $\tau$ is close to zero or one. In an extreme case, a proportion such as $C_{T}(\tau, \tau)$ may even be zero or one when the sample size is small. The simulation experiments therefore do not include $\tau=0.05$.

(A) The observations are generated from a bivariate Gaussian distribution with time varying correlation

$$
\rho_{t}=\frac{1-e^{\mu_{t}}}{1+e^{\mu_{t}}}, \quad t=1, \ldots, T
$$

where $\mu_{t}$ is a random walk process obtained from Gaussian innovations with

mean zero and variance equal to $q^{2}$. The logistic transform guarantees that 
$\rho_{t} \in(-1,1)$. We consider samples of $T=200$ and 400 observations, with $q$ taking values $0, .1, .25, .5$.

The upper part of Table 1 contains the rejection frequencies of the biquantic stationarity tests and the Box-Ljung bi-quantilogram Q-tests for the four quadrants $B B, A A, B A, A B$. The column $q=0$ provides the empirical size of the tests; this appears to be already well under control in a sample of $T=200$ observations. As regards power (provided by the simulated rejection frequencies for $q>0$ ), this is a maximum at $\tau=.5$, where it appears to be the same in all quadrants. The lower quadrant test, $B B$, is more powerful for $\tau<0.5$, that is in the lower tail of the distribution, while the upper quadrant test, $A A$, is more powerful in the upper tail $(\tau>0.5)$. Furthermore the power for $B B$ at $\tau$ is the same as the power for $A A$ at $1-\tau$; this is a reflection of the symmetry properties of the Gaussian distribution.

The table shows that the bi-quantic tests are significantly more powerful than the Box-Ljung bi-quantilogram Q-tests. The latter have been computed with $m=5$ autocorrelation coefficients, but power would not increase much if $m=10$ (while it would considerably reduce for $m=1$ or 2 ). For both tests power is considerably higher for $T=400$ as opposed to $T=200$, thereby providing an indication of the consistency of the tests against this 
data generating process.

The lower part of the table contains the results for the combined test, $\eta_{\tau}(B Q ; 3)$, the quadrant association test, $\eta_{\tau}(B Q+\overline{B Q})$, and the test of changing correlation. All of them appear to be more powerful than the single quadrant bi-quantic tests. The quadrant association test appears to be more powerful than the combined test for $\tau=0.50$. For small values of $q$ the maximum power is attained by the test of changing correlation, which is not unexpected reasonable since correlation is an appropriate measure of dependence for Gaussian observations. However, the quadrant association test is better for $q=0.25$ and above.

(B) The observations are generated from a bivariate Gaussian distribution with a structural break in the correlation coefficient. We set $\rho_{t}=.75$ in the first half of the sample and $\rho_{t}=.75, .50, .25,0$ in the second half. For brevity we only report rejection frequencies for a sample size of $T=300$. Furthermore, given that the same symmetry properties hold as in case (A), we do not provide results for the $A A$ and $A B$ quadrants. Table 2 contains all the relevant results. Again the copula bi-quantic tests are significantly more powerful than those based on the bi-quantilogram, but the differences appear to be even greater. Overall, the test of changing correlation appears to display 
the highest power, although when $\rho_{t}=.5$ in the second half of the sample the quadrant association test rejects the null hypothesis most frequently. Broadly speaking, these results for a structural break in correlation are qualitatively the same as those obtained in case (A).

(C) Table 3 presents the results for the case of a Clayton copula with structural break in the dependence parameter. In order to simulate from a bivariate Clayton copula we make independent draws from a uniform $(0,1)$ distribution to give $u_{1 t}$ and $v_{t}$, and then set

$$
u_{2 t}=\left(\left(v_{t}^{-\theta /(1+\theta)}-1\right) u_{1 t}^{-\theta}+1\right)^{-1 / \theta}, t=1, \ldots, T ;
$$

see Embrechts et al (2003, p9). We consider sample sizes of $T=200$ and 400 observations, setting $\theta=1$ in the first half of the sample and $\theta=1,2.5,7.5,15$ in the second half. Since Kendall's Tau is $\theta /(\theta+2)$, there is more dependence after the break.

The results show that there is no longer a symmetry in the $B B$ and $A A$ tests, though the $B A$ and $A B$ tests do, as before, have similar power. In fact these tests are now more powerful than the the $B B$ and $A A$ tests. As in the previous cases the bi-quantilogram tests are significantly less powerful. 
The quadrant association test appears to be the most powerful test overall, performing significantly better than the combined bi-quantic tests. The test of changing correlation has very low power. Different marginals would give different results for the changing correlation test, but unless the joint distribution is bivariate normal, it seems that a good performance cannot be guaranteed.

Taking all three sets of results together, the quadrant association test at $\tau=0.5$ is clearly the one to be recommended for detecting overall changes in dependence. It also seems to be the preferred test for other values of $\tau$.

\section{Correcting for time variation in the indi- vidual series}

If the quantiles in the individual series change slowly over time, the tests will tend to reject even if the copula is constant. A nonparametric correction for serial correlation will not be effective even for stationary movements if they are highly persistent.

If the movements in quantiles are due solely to changes in volatility, the series may be adjusted by dividing by a measure of dispersion. A stochastic 
volatility (SV) model can be fitted to each of the series and the resulting (two sided) smoothed estimates of the standard deviations used to rescale the observations; Patton (2006, p536-7) and van den Goorbergha, Genest and Werkerc (2005) use GARCH, a one-sided filter. Note that if the estimation of the SV model is based on quasi-maximum likelihood (QML), the effect of heavy-tails is mitigated by the taking of logarithms (of the squared observations); see Harvey et al. (1994).

For tests based only on bi-quantics at $\tau=0.5$, there is no need to prefilter if the medians are constant. Indeed if the long-run variance correction is employed, the medians can be time-varying provided they are stationary. The assumption of constant medians is plausible for exchange rate returns, but less so for stock returns.

We provide Monte Carlo simulation results for a bivariate SV data generating process, estimated by QML. Bivariate Gaussian series were first generated, as was done for constructing tables 1,2 and 3, and then each series was multiplied by a time-varying standard deviation obtained from independent SV models.

Tables 4a,b and c reports the simulated rejection frequencies for the copula based tests and the changing correlation test, run at $5 \%$ significance 
level, for a sample size of $T=400$ or $T=200$. For brevity we only present results for the case $\sigma_{\kappa}=0.025$. The left hand panel of the table reports the rejection frequencies of the tests applied to the raw series, $y_{1 t}$ and $y_{2 t}$. The right hand panel of the table show the results after rescaling the series by estimates of the unobserved time-varying standard deviation, that is $y_{i t} / \exp \left(0.5 \widehat{h}_{i t}\right), t=1, . ., T, i=1,2$. The $\widehat{h}_{i t}^{\prime} s$ are obtained by applying the state space smoother to the log of squared observations (for each series in turn), with parameters obtained by QML estimation of a random walk plus noise model.

As expected, for $\tau=0.5$ there is no need to estimate volatility; on the other hand no power loss is induced by estimation. For the other values of $\tau$, ignoring time-varying volatility makes the tests oversized. The volatility correction brings the empirical size very close to the nominal level of $5 \%$; the power properties of the tests are qualitatively very similar to those observed in tables 1,2 and 3.

Tests for changing correlation will be affected by changes in the distribution. Prefiltering to remove changing volatility may not be sufficient to ensure that the sizes of the tests are close to the nominal if there are other movements in the marginal distributions, for example in kurtosis. In this 
case the time-varying $\tau$-quantiles must be estimated directly, possibly as in Yu and Jones (1998), De Rossi and Harvey (2006, 2008) or Harvey (2008), and the bi-quantics formed from them.

\section{Application to financial contagion}

As an illustration, we apply our tests to the daily returns of stock market indices of two East Asian countries, Korea and Thailand, over the period 1995-1999. The sample includes the financial turmoil period of summerautumn 1997 (the "Asian crisis") characterized by huge losses in the Asian markets. In the 4 months period August-November, the Korean and Thai stock indices fell by over $40 \%$. There were few repercussions in the United States and Europe.

The financial contagion issue is whether market interdependence becomes more acute in times of financial turmoils. In particular, do negative shocks from one area transmit more intensely to the other markets ? This is sometimes called "correlation breakdown", denoting a statistically significant increase in correlation during the crisis period. Forbes and Rigobon (2002) pointed out that volatility increases during crises and argued that this should 
be taken into account when computing the tests of financial contagion. After adjusting for heteroskedasticity they found no evidence of correlation breakdown during the Asian crisis. However, Rodriguez (2007) analysed contagion by estimating time-varying ("switching") volatilities and copulas across markets and found evidence of changing dependence structures (including tail dependence) during periods of financial turmoil. The most notable example was the Korea-Thailand case.

The test statistics were computed for $\tau=0.25,0.50$ and 0.75 , with bandwidth set to $m=8$, obtained from a bandwidth rule of $m=4(T / 100)^{1 / 4}$. To account for changing volatility, the observations were standardized by dividing by smoothed standard deviations from stochastic volatility models estimated by QML. The stationarity test statistics for $\tau=0.25,0.50$ and 0.75 were, respectively, $.24, .26$ and .21 for Thailand and $.19, .43$ and .57 for Korea. Thus there is some evidence that the volatility correction has not been completely successful for Korea as the test statistic for $\tau=0.75$ is statistically significant at the $5 \%$ level. The quadrant association and combined bi-quantic tests for $\tau=0.5$ gave rejections at the $1 \%$ level of significance, taking values of 1.67 and 2.37 respectively. The combined tests for $\tau=0.25$ and 0.75 also rejected at the $1 \%$ level of significance, with values of 1.04 
and 1.42. Lower and upper tail statistics, $\eta_{0.25}(B Q ; B B)$ and $\eta_{0.75}(B Q ; A A)$, were 0.68 and 1.19 respectively, the former significant at the $1 \%$ level of significance, the latter at the $5 \%$ level. The idea of using single tail tests was to isolate movements in each tail in the event of asymmetry.

\section{Conclusion}

The proposed indicator stationarity tests appear to have good power properties against slowly changing dependence and sudden breaks. The preferred test is the quadrant association test. Simulations indicate that the tests are still effective if pre-filtering is carried out to correct for changing volatility or, more generally, changing quantiles. Tests based on medians seem to have the highest power overall and if the medians can be assumed constant, or even stationary, no pre-filtering is necessary. ${ }^{2}$

The tests were applied to daily returns of stock market indices in Korea and Thailand over the period 1995-1999. The hypothesis of a constant copula is strongly rejected by tests at the median and at the two quartiles.

Unstable relationships between assets has serious consequences for port-

\footnotetext{
${ }^{2}$ Note, however, that while the means of stock prices can usually be taken to be constant, the null hypothesis of a stationary median is often rejected.
} 
folio selection. If time variation is found in the relationship between variables then we might try to model it. This seems to be a topic on which more work could be done. It is also worth investigating if and how a relationship might change in different parts of the copula and how the individual quadrant tests might detect such changes.

\section{APPENDICES}

\section{A Correlations between bivariate quantile in-}

\section{dicators}

Assumption Suppose the two time series, $y_{1 t}, y_{2 t}$, are serially independent with a time-invariant joint distribution (ie $y_{1 t}, y_{2 t}$ are independently and $i d e n-$ tically distributed), such that the marginal distributions are continuous and positive.

To find the covariance between the bivariate $\tau$-quantile indicators the appropriate products and probabilities must be evaluated. Thus for $B I Q\left(y_{1 t} \leq\right.$

$\left.\xi_{1}(\tau), y_{2 t} \leq \xi_{2}(\tau)\right)$ and $B I Q\left(y_{1 t}>\xi_{1}(\tau), y_{2 t}>\xi_{2}(\tau)\right)$ we have the following: (i) when $y_{1 t} \leq \xi_{1}\left(\tau_{1}\right)$ and $y_{2 t} \leq \xi_{2}\left(\tau_{2}\right)$, the product $(C(\tau, \tau)-1)(1-2 \tau+$ $C(\tau, \tau)$ ) is weighted by $C(\tau, \tau)$; (ii) when $y_{1 t}>\xi_{1}\left(\tau_{1}\right)$ and $y_{2 t}>\xi_{2}\left(\tau_{2}\right)$, 
the product $C(\tau, \tau)(C(\tau, \tau)-2 \tau)$ is weighted by $1-2 \tau+C(\tau, \tau)$; and when $y_{1 t}>\xi_{1}\left(\tau_{1}\right)$ and $y_{2 t} \leq \xi_{2}\left(\tau_{2}\right)$, or $y_{1 t} \leq \xi_{1}\left(\tau_{1}\right)$ and $y_{2 t}>\xi_{2}\left(\tau_{2}\right)$, the product $C(\tau, \tau)(1-2 \tau+C(\tau, \tau))$ is weighted by $C(\tau, \tau)-\tau$. On collecting terms we find that the covariance between $B I Q\left(y_{1 t} \leq \xi_{1}(\tau), y_{2 t} \leq \xi_{2}(\tau)\right)$ and $B I Q\left(y_{1 t}>\xi_{1}(\tau), y_{2 t}>\xi_{2}(\tau)\right)$ is

$$
-C(\tau, \tau)(1-2 \tau+C(\tau, \tau))
$$

The correlation in (8) is found by dividing by the two standard deviations.

\section{B Asymptotic distribution}

Suppose assumption in appendix A holds and that the copula has continuous partial derivatives. Then

$$
\eta_{\tau}(B Q) \stackrel{d}{\rightarrow} \int_{0}^{1}(W(r)-r W(1))^{2} d r \equiv C v M
$$

where $\eta_{\tau}(B Q)$ is the test statistic in (9) and $W(r)$ is a standard Wiener process.

PROOF: 
Rewrite the bivariate $\tau$-quantic, (4), as

$$
B I Q_{t}(\tau)=-\left(A_{t, T}+B_{t, T}+C_{T}^{\dagger}(\tau, \tau)-C_{T}(\tau, \tau)\right)
$$

where

$$
\begin{aligned}
A_{t, T} & =1\left(y_{1 t} \leq \xi_{1}(\tau), y_{2 t} \leq \xi_{2}(\tau)\right)-C_{T}^{\dagger}(\tau, \tau), \\
B_{t, T} & =1\left(y_{1 t} \leq \widetilde{\xi}_{1}(\tau), y_{2 t} \leq \widetilde{\xi}_{2}(\tau)\right)-1\left(y_{1 t} \leq \xi_{1}(\tau), y_{2 t} \leq \xi_{2}(\tau)\right), \\
C_{T}^{\dagger}(\tau, \tau) & =\frac{1}{T} \sum_{t=1}^{T} 1\left(y_{1 t} \leq \xi_{1}(\tau), y_{2 t} \leq \xi_{2}(\tau)\right), \\
C_{T}(\tau, \tau) & =\frac{1}{T} \sum_{t=1}^{T} 1\left(y_{1 t} \leq \widetilde{\xi}_{1}(\tau), y_{2 t} \leq \widetilde{\xi}_{2}(\tau)\right) .
\end{aligned}
$$

Consider first the term $A_{t, T}$. Under the i.i.d. hypothesis, $1\left(y_{1 t} \leq \xi_{1}(\tau), y_{2 t} \leq\right.$ $\left.\xi_{2}(\tau)\right)$ is a sequence of independent Bernoulli random variables with mean $C(\tau, \tau)$, the true copula evaluated at $\tau, \tau$, and variance $V=C(\tau, \tau)(1-$ $C(\tau, \tau))$; the quantity $C_{T}^{\dagger}(\tau, \tau)$ is the sample mean. Therefore the invariance principle holds and so, for $r \in[0,1]$,

$$
V^{-\frac{1}{2}} T^{-\frac{1}{2}} \sum_{t=1}^{[T r]} A_{t, T} \Rightarrow W(r)-r W(1)
$$


a standard Brownian bridge, where $\Rightarrow$ denotes 'converges weakly'.

The second step is to show that, after taking scaled partial sums, the two terms $B_{t, T}$ and $C_{T}^{\dagger}(\tau, \tau)-C_{T}(\tau, \tau)$ converge both to zero in probability. Under continuity of the marginals and continuous partial derivatives of the copula, Fermanian et al. (2004) establish weak convergence of the empirical copula process $\sqrt{T}\left(C_{T}(.,)-.C(.,).\right)$, which implies stochastic equicontinuity. Thus, as $\widetilde{\xi}_{i}(\tau) \stackrel{p}{\rightarrow} \xi_{i}(\tau), i=1,2$, we have that $\sqrt{T}\left(C_{T}^{\dagger}(\tau, \tau)-C_{T}(\tau, \tau)\right)=o_{p}(1)$; see also van der Vaart and Wellner $(1996$ p.389). Similarly,

$$
\begin{aligned}
\frac{1}{\sqrt{T}} \sum_{t=1}^{[T r]} B_{t, T} & =\sqrt{r} \frac{1}{\sqrt{T r}} \sum_{t=1}^{[T r]}\left(1\left(y_{1 t} \leq \widetilde{\xi}_{1}(\tau), y_{2 t} \leq \widetilde{\xi}_{2}(\tau)\right)-1\left(y_{1 t} \leq \xi_{1}(\tau), y_{2 t} \leq \xi_{2}(\tau)\right)\right) \\
& =\sqrt{r} \frac{[T r]}{\sqrt{T r}}\left(C_{[T r]}(\tau, \tau)-C_{[T r]}^{\dagger}(\tau, \tau)\right)=o_{p}(1) .
\end{aligned}
$$

Therefore, by an application of the continuous mapping theorem, the Cramérvon Mises distribution in (13) is obtained.

Acknowledgments Earlier versions of this paper were presented at $C R E$ ATES in Aarhus, Alicante, CEMFI (Madrid), the Cambridge Econometrics workshop and the conference on "Measuring dependence in economics and finance" at CASS (London) in December 200\%. We are grateful to Pe- 
ter Christoffersen, Mardi Dungey, Neils Haldrup, Hashem Pesaran, Alessio Sancetta, Richard Smith, Robert Taylor and others for helpful comments. Any errors are our responsibility. We acknowledge financial support from the ESRC under the grant Time-Varying Quantiles, RES-062-23-0129.

\section{REFERENCES}

Busetti, F. and A. C. Harvey (2007) Tests of time-invariance based on quantile indicators. Cambridge, Faculty of Economics, CWPE 0657

Cherubini, U., Luciano, E. and W. Vecchiato (2004). Copula methods in Finance. John Wiley and Sons: Chichester.

Das, S. and R. Upal. (2004) Systematic risk and international portfolio choice. Journal of Finance, 59, 2809-34.

De Jong, R.M., Amsler, C. and P. Schmidt (2007). A Robust Version of the KPSS Test based on Indicators, Journal of Econometrics, 137, 311-33.

De Rossi, G. and Harvey, A C (2006). Time-varying quantiles. Cambridge, Faculty of Economics, CWPE 0649.

De Rossi, G. and Harvey, A C (2008). Quantiles, expectiles and splines. Forthcoming in Journal of Econometrics.

Doornik, J. A. (1999). Ox: An Object-Oriented Matrix Language. 3rd edition. Timberlake Consultants Press: London. 
Embrechts P, Lindskog F, and A.J. McNeil (2003) Modelling dependence with copulas and applications to risk management. In: Handbook of Heavy Tailed Distributions in Finance, ed. S. Rachev, Elsevier, Chapter 8, pp. $329-384$

Engle, R.F., and Manganelli, S. (2004). CAViaR: conditional autoregressive value at risk by regression quantiles, Journal of Business and Economic Statistics, 22, 367-381.

Fermanian, J-D, Radulovic, D. and M. Wegkamp (2004). Weak convergence of empirical copula processes. Bernouilli, 10, 847-60.

Forbes, K. and R. Rigobon (2002). No contagion, only interdependence: measuring stock market movements. Journal of Finance, 57, 2223-61.

Harvey, A.C. (2008). Dynamic distributions and changing copulas. Mimeo.

Harvey A.C., E. Ruiz and N. Shephard (1994). Multivariate stochastic variance models. Review of Economic Studies 61, 247-64.

Harvey, A. C., and M. Streibel (1998). Testing for a slowly changing level with special reference to stochastic volatility. Journal of Econometrics 87, $167-89$.

Koopman, S.J., N. Shephard and J. Doornik (1999). Statistical algorithms for models in state space using SsfPack 2.2. Econometrics Journal, 
$2,113-66$.

Kruskal, W.H. (1958). Ordinal measures of association. Journal of the American Statistical Association, 53, 814-61.

Kwiatkowski, D., Phillips, P.C.B., Schmidt P., and Y.Shin (1992). Testing the null hypothesis of stationarity against the alternative of a unit root: How sure are we that economic time series have a unit root? Journal of Econometrics 44, 159-78.

Linton, O. and Y.-J.Whang (2007). The quantilogram: with an application to evaluating directional predictability. Journal of Econometrics, 141, $250-82$.

McNeil, A.J., Frey, R. and P.Embrechts (2005). Quantitative Risk Management. Princeton Series in Finance.

Nyblom, J.(1989). Testing for the constancy of parameters over time. Journal of the American Statistical Association 84, 223-30.

Nyblom, J., and A.C. Harvey (2001). Testing against smooth stochastic trends, Journal of Applied Econometrics, 16, 415-29.

Nyblom, J. and T. Mäkeläinen (1983). Comparison of tests for the presence of random walk coefficients in a simple linear model, Journal of the American Statistical Association, 78, 856-64. 
Patton, A. J. (2006). Modelling asymmetric exchange rate dependence. International Economic Review, 47, 527-56.

Rodriguez, J.C. (2007). Measuring financial contagion: a copula approach. Journal of Empirical Finance, 14, 401-23.

Van den Goorbergha, R.W.J., Genest, C. and B.J.M. Werkerc (2005). Bivariate option pricing using dynamic copula models, Insurance: Mathematics and Economics 37, 101-114.

Van der Vaart, A.W. and Wellner, J.A. (1996) Weak Convergence and Empirical Processes. New York: Springer-Verlag.

Yu, K. and M.C. Jones (1998), Local linear quantile regression, Journal of the American Statistical Association, 93, 228-37. 
Table 1: Gaussian Copula with time-varying correlation: simulated rejection frequencies of the tests

\begin{tabular}{|c|c|c|c|c|c|c|c|c|c|c|}
\hline & \multirow[b]{2}{*}{ tau } & \multicolumn{5}{|c|}{$T=200$} & \multicolumn{4}{|c|}{$T=400$} \\
\hline & & $q$ & 0 & 0.1 & 0.25 & 0.5 & 0 & 0.1 & 0.25 & 0.5 \\
\hline \multicolumn{11}{|l|}{ Bi-quantic test } \\
\hline \multirow{5}{*}{ BB } & 0.10 & & .02 & .06 & .13 & .17 & .04 & .18 & .30 & .36 \\
\hline & 0.25 & & .05 & .18 & .35 & .42 & .05 & .42 & .60 & .63 \\
\hline & 0.50 & & .05 & .18 & .41 & .56 & .05 & .41 & .68 & .76 \\
\hline & 0.75 & & .05 & .08 & .15 & .20 & .05 & .16 & .28 & .34 \\
\hline & 0.90 & & .05 & .06 & .08 & .09 & .05 & .08 & .12 & .15 \\
\hline \multirow{5}{*}{ AA } & 0.10 & & .05 & .06 & .08 & .10 & .05 & .08 & .13 & .15 \\
\hline & 0.25 & & .05 & .08 & .15 & .20 & .05 & .16 & .28 & .35 \\
\hline & 0.50 & & .05 & .18 & .42 & .56 & .05 & .41 & .68 & .76 \\
\hline & 0.75 & & .05 & .19 & .35 & .42 & .05 & .42 & .60 & .63 \\
\hline & 0.90 & & .03 & .06 & .13 & .17 & .04 & .18 & .30 & .36 \\
\hline \multirow{5}{*}{$\mathrm{BA}$} & 0.10 & & .05 & .07 & .13 & .19 & .05 & .11 & .25 & .33 \\
\hline & 0.25 & & .05 & .11 & .25 & .36 & .05 & .24 & .45 & .55 \\
\hline & 0.50 & & .05 & .18 & .42 & .56 & .05 & .41 & .68 & .76 \\
\hline & 0.75 & & .05 & .11 & .25 & .36 & .05 & .24 & .44 & .55 \\
\hline & 0.90 & & .05 & .07 & .13 & .19 & .05 & .12 & .25 & .33 \\
\hline \multirow{5}{*}{$A B$} & 0.10 & & .05 & .07 & .13 & .19 & .05 & .12 & .25 & .33 \\
\hline & 0.25 & & .05 & .11 & .25 & .36 & .05 & .24 & .44 & .55 \\
\hline & 0.50 & & .05 & .18 & .42 & .56 & .05 & .41 & .68 & .76 \\
\hline & 0.75 & & .05 & .11 & .25 & .36 & .05 & .24 & .45 & .55 \\
\hline & 0.90 & & .05 & .07 & .13 & .19 & .05 & .11 & .25 & .33 \\
\hline \multicolumn{11}{|l|}{ Bi-quantilogram test } \\
\hline \multirow{5}{*}{ BB } & 0.10 & & .07 & .09 & .11 & .14 & .09 & .11 & .16 & .20 \\
\hline & 0.25 & & .05 & .10 & .18 & .26 & .05 & .15 & .32 & .45 \\
\hline & 0.50 & & .05 & .06 & .15 & .34 & .05 & .10 & .37 & .61 \\
\hline & 0.75 & & .05 & .05 & .06 & .07 & .05 & .06 & .07 & .10 \\
\hline & 0.90 & & .05 & .05 & .05 & .05 & .05 & .05 & .05 & .06 \\
\hline \multirow{5}{*}{ AA } & 0.10 & & .05 & .05 & .05 & .05 & .05 & .05 & .06 & .06 \\
\hline & 0.25 & & .05 & .05 & .06 & .07 & .05 & .05 & .07 & .10 \\
\hline & 0.50 & & .05 & .06 & .16 & .34 & .05 & .10 & .37 & .61 \\
\hline & 0.75 & & .05 & .10 & .18 & .27 & .05 & .16 & .33 & .46 \\
\hline & 0.90 & & .07 & .09 & .11 & .14 & .10 & .11 & .16 & .21 \\
\hline \multirow{5}{*}{ BA } & 0.10 & & .05 & .05 & .08 & .12 & .05 & .06 & .11 & .17 \\
\hline & 0.25 & & .05 & .06 & .11 & .20 & .05 & .07 & .20 & .36 \\
\hline & 0.50 & & .05 & .06 & .15 & .34 & .05 & .10 & .37 & .61 \\
\hline & 0.75 & & .05 & .05 & .10 & .19 & .05 & .08 & .20 & .36 \\
\hline & 0.90 & & .04 & .05 & .08 & .11 & .05 & .06 & .11 & .17 \\
\hline \multirow{5}{*}{$A B$} & 0.10 & & .05 & .05 & .07 & .12 & .05 & .06 & .10 & .17 \\
\hline & 0.25 & & .05 & .06 & .10 & .20 & .05 & .07 & .19 & .36 \\
\hline & 0.50 & & .05 & .06 & .15 & .34 & .05 & .10 & .37 & .61 \\
\hline & 0.75 & & .05 & .05 & .10 & .20 & .05 & .07 & .20 & .35 \\
\hline & 0.90 & & .05 & .05 & .08 & .12 & .05 & .06 & .11 & .18 \\
\hline \multirow[t]{5}{*}{ Combined bi-quantic test } & 0.10 & & .04 & .07 & .19 & .28 & .04 & .19 & .39 & .51 \\
\hline & 0.25 & & .05 & .20 & .43 & .56 & .05 & .46 & .69 & .76 \\
\hline & 0.50 & & .05 & .29 & .64 & .80 & .05 & .59 & .87 & .95 \\
\hline & 0.75 & & .05 & .20 & .43 & .55 & .05 & .46 & .69 & .76 \\
\hline & 0.90 & & .04 & .07 & .19 & .29 & .05 & .20 & .39 & .51 \\
\hline \multirow[t]{5}{*}{ Quadrant association test } & 0.10 & & .05 & .09 & .20 & .30 & .05 & .17 & .36 & .47 \\
\hline & 0.25 & & .05 & .20 & .41 & .54 & .05 & .41 & .62 & .70 \\
\hline & 0.50 & & .05 & .38 & .73 & .87 & .05 & .68 & .92 & .97 \\
\hline & 0.75 & & .05 & .20 & .41 & .54 & .05 & .41 & .62 & .70 \\
\hline & 0.90 & & .05 & .09 & .20 & .30 & .05 & .18 & .36 & .48 \\
\hline Changing correlation test & & & .05 & .50 & .67 & .67 & .05 & .77 & .82 & .77 \\
\hline
\end{tabular}


Table 2: Gaussian Copula with structural breaks in correlation: simulated rejection frequencies ( $T=300)$

\begin{tabular}{|c|c|c|c|c|c|c|}
\hline & tau & rho & $(0.75,0.75)$ & $(0.75,0.50)$ & $(0.75,0.25)$ & $(0.75,0)$ \\
\hline \multicolumn{7}{|l|}{ Bi-quantic test } \\
\hline & 0.10 & & .05 & .10 & .26 & .46 \\
\hline & 0.25 & & .05 & .14 & .38 & .68 \\
\hline \multirow[t]{5}{*}{ BB } & 0.50 & & .05 & .13 & .34 & .62 \\
\hline & 0.75 & & .05 & .10 & .21 & .35 \\
\hline & 0.90 & & .05 & .07 & .11 & .14 \\
\hline & 0.10 & & .05 & .09 & .16 & .23 \\
\hline & 0.25 & & .05 & .15 & .36 & .58 \\
\hline \multirow[t]{3}{*}{ BA } & 0.50 & & .05 & .20 & .51 & .78 \\
\hline & 0.75 & & .05 & .16 & .37 & .58 \\
\hline & 0.90 & & .05 & .10 & .17 & .24 \\
\hline \multicolumn{7}{|l|}{ Bi-quantilogram test } \\
\hline & 0.10 & & .06 & .07 & .11 & .15 \\
\hline & 0.25 & & .05 & .05 & .07 & .13 \\
\hline \multirow[t]{5}{*}{ BB } & 0.50 & & .05 & .05 & .07 & .09 \\
\hline & 0.75 & & .05 & .06 & .06 & .06 \\
\hline & 0.90 & & .04 & .05 & .05 & .05 \\
\hline & 0.10 & & .06 & .06 & .06 & .07 \\
\hline & 0.25 & & .05 & .05 & .07 & .10 \\
\hline \multirow[t]{3}{*}{ BA } & 0.50 & & .05 & .05 & .08 & .15 \\
\hline & 0.75 & & .05 & .05 & .07 & .11 \\
\hline & 0.90 & & .06 & .06 & .06 & .07 \\
\hline \multirow[t]{5}{*}{ Combined bi-quantic test } & 0.10 & & .05 & .12 & .32 & .54 \\
\hline & 0.25 & & .05 & .22 & .62 & .91 \\
\hline & 0.50 & & .05 & .28 & .76 & .97 \\
\hline & 0.75 & & .05 & .22 & .63 & .91 \\
\hline & 0.90 & & .05 & .13 & .31 & .54 \\
\hline \multirow[t]{5}{*}{ Quadrant association test } & 0.10 & & .05 & .14 & .31 & .45 \\
\hline & 0.25 & & .05 & .29 & .71 & .93 \\
\hline & 0.50 & & .05 & .42 & .89 & .99 \\
\hline & 0.75 & & .05 & .31 & .72 & .93 \\
\hline & 0.90 & & .05 & .15 & .31 & .46 \\
\hline Changing correlation test & & & .05 & .37 & .93 & 1.00 \\
\hline
\end{tabular}


Table 3: Clayton Copula with a structural break in dependence: simulated rejection frequencies of the tests

\begin{tabular}{|c|c|c|c|c|c|c|c|c|c|c|}
\hline & \multirow[b]{2}{*}{ tau } & \multicolumn{5}{|c|}{$T=200$} & \multicolumn{4}{|c|}{$T=400$} \\
\hline & & theta & $(1,1)$ & $(1,2.5)$ & $(1,7.5)$ & $(1,15)$ & $(1,1)$ & $(1,2.5)$ & $(1,7.5)$ & $(1,15)$ \\
\hline \multicolumn{11}{|l|}{ Bi-quantic test } \\
\hline & 0.10 & & .05 & .08 & .14 & .16 & .05 & .13 & .25 & .29 \\
\hline & 0.25 & & .05 & .12 & .27 & .32 & .05 & .20 & .49 & .57 \\
\hline \multirow[t]{5}{*}{ BB } & 0.50 & & .05 & .12 & .35 & .45 & .05 & .19 & .60 & .75 \\
\hline & 0.75 & & .05 & .08 & .22 & .34 & .05 & .10 & .38 & .58 \\
\hline & 0.90 & & .05 & .06 & .08 & .13 & .05 & .06 & .11 & .21 \\
\hline & 0.10 & & .05 & .07 & .10 & .11 & .05 & .09 & .17 & .20 \\
\hline & 0.25 & & .05 & .09 & .20 & .24 & .05 & .15 & .37 & .45 \\
\hline \multirow[t]{5}{*}{ AA } & 0.50 & & .05 & .12 & .35 & .44 & .05 & .20 & .60 & .75 \\
\hline & 0.75 & & .05 & .10 & .35 & .51 & .05 & .17 & .63 & .82 \\
\hline & 0.90 & & .04 & .06 & .16 & .29 & .04 & .08 & .33 & .58 \\
\hline & 0.10 & & .05 & .11 & .27 & .34 & .05 & .19 & .55 & .68 \\
\hline & 0.25 & & .05 & .19 & .60 & .73 & .05 & .34 & .88 & .95 \\
\hline \multirow[t]{5}{*}{ BA } & 0.50 & & .05 & .19 & .72 & .88 & .05 & .35 & .95 & .99 \\
\hline & 0.75 & & .05 & .10 & .46 & .73 & .05 & .15 & .75 & .95 \\
\hline & 0.90 & & .05 & .06 & .13 & .25 & .05 & .06 & .20 & .45 \\
\hline & 0.10 & & .05 & .11 & .27 & .34 & .05 & .20 & .55 & .68 \\
\hline & 0.25 & & .05 & .18 & .59 & .73 & .05 & .34 & .88 & .95 \\
\hline \multirow[t]{3}{*}{$A B$} & 0.50 & & .05 & .19 & .72 & .88 & .05 & .34 & .95 & .99 \\
\hline & 0.75 & & .05 & .10 & .46 & .73 & .05 & .15 & .74 & .95 \\
\hline & 0.90 & & .05 & .05 & .12 & .24 & .05 & .06 & .20 & .44 \\
\hline \multicolumn{11}{|l|}{ Bi-quantilogram test } \\
\hline \multirow{5}{*}{ BB } & 0.10 & & .06 & .06 & .06 & .07 & .06 & .06 & .06 & .07 \\
\hline & 0.25 & & .05 & .05 & .07 & .07 & .05 & .06 & .07 & .08 \\
\hline & 0.50 & & .05 & .05 & .07 & .08 & .05 & .05 & .07 & .10 \\
\hline & 0.75 & & .06 & .05 & .06 & .06 & .05 & .05 & .06 & .07 \\
\hline & 0.90 & & .05 & .05 & .06 & .05 & .05 & .05 & .05 & .06 \\
\hline \multirow{5}{*}{ AA } & 0.10 & & .05 & .05 & .05 & .05 & .04 & .05 & .05 & .05 \\
\hline & 0.25 & & .05 & .05 & .05 & .06 & .05 & .06 & .06 & .07 \\
\hline & 0.50 & & .05 & .05 & .07 & .07 & .05 & .06 & .08 & .11 \\
\hline & 0.75 & & .05 & .05 & .08 & .10 & .04 & .05 & .10 & .14 \\
\hline & 0.90 & & .12 & .13 & .13 & .14 & .09 & .09 & .11 & .14 \\
\hline \multirow{5}{*}{ BA } & 0.10 & & .07 & .10 & .18 & .21 & .06 & .09 & .17 & .21 \\
\hline & 0.25 & & .05 & .07 & .17 & .23 & .05 & .08 & .24 & .34 \\
\hline & 0.50 & & .05 & .06 & .19 & .32 & .05 & .06 & .28 & .50 \\
\hline & 0.75 & & .05 & .05 & .11 & .21 & .05 & .06 & .14 & .30 \\
\hline & 0.90 & & .04 & .05 & .06 & .09 & .04 & .05 & .06 & .10 \\
\hline \multirow{5}{*}{$\mathrm{AB}$} & 0.10 & & .06 & .10 & .17 & .20 & .06 & .09 & .17 & .20 \\
\hline & 0.25 & & .04 & .06 & .16 & .22 & .05 & .08 & .24 & .34 \\
\hline & 0.50 & & .05 & .06 & .19 & .32 & .05 & .06 & .28 & .50 \\
\hline & 0.75 & & .04 & .05 & .11 & .21 & .05 & .05 & .14 & .30 \\
\hline & 0.90 & & .05 & .05 & .06 & .09 & .04 & .05 & .06 & .10 \\
\hline \multirow[t]{5}{*}{ Combined bi-quantic test } & 0.10 & & .04 & .13 & .37 & .46 & .05 & .26 & .79 & .91 \\
\hline & 0.25 & & .05 & .25 & .82 & .94 & .05 & .51 & .99 & 1.00 \\
\hline & 0.50 & & .05 & .26 & .93 & .99 & .05 & .50 & 1.00 & 1.00 \\
\hline & 0.75 & & .05 & .13 & .70 & .95 & .05 & .24 & .96 & 1.00 \\
\hline & 0.90 & & .05 & .06 & .20 & .44 & .05 & .08 & .41 & .81 \\
\hline \multirow[t]{5}{*}{ Quadrant association test } & 0.10 & & .05 & .18 & .51 & .62 & .05 & .35 & .86 & .95 \\
\hline & 0.25 & & .05 & .36 & .91 & .97 & .05 & .64 & 1.00 & 1.00 \\
\hline & 0.50 & & .05 & .39 & .98 & 1.00 & .05 & .67 & 1.00 & 1.00 \\
\hline & 0.75 & & .05 & .18 & .80 & .98 & .05 & .30 & .98 & 1.00 \\
\hline & 0.90 & & .05 & .06 & .21 & .46 & .05 & .08 & .37 & .76 \\
\hline Changing correlation test & & & .05 & .08 & .14 & .15 & .05 & .12 & .25 & .28 \\
\hline
\end{tabular}


Table 4a: Time varying gaussian copula, with stochastic volatility. Simulated rejection frequencies of the tests: $T=400$

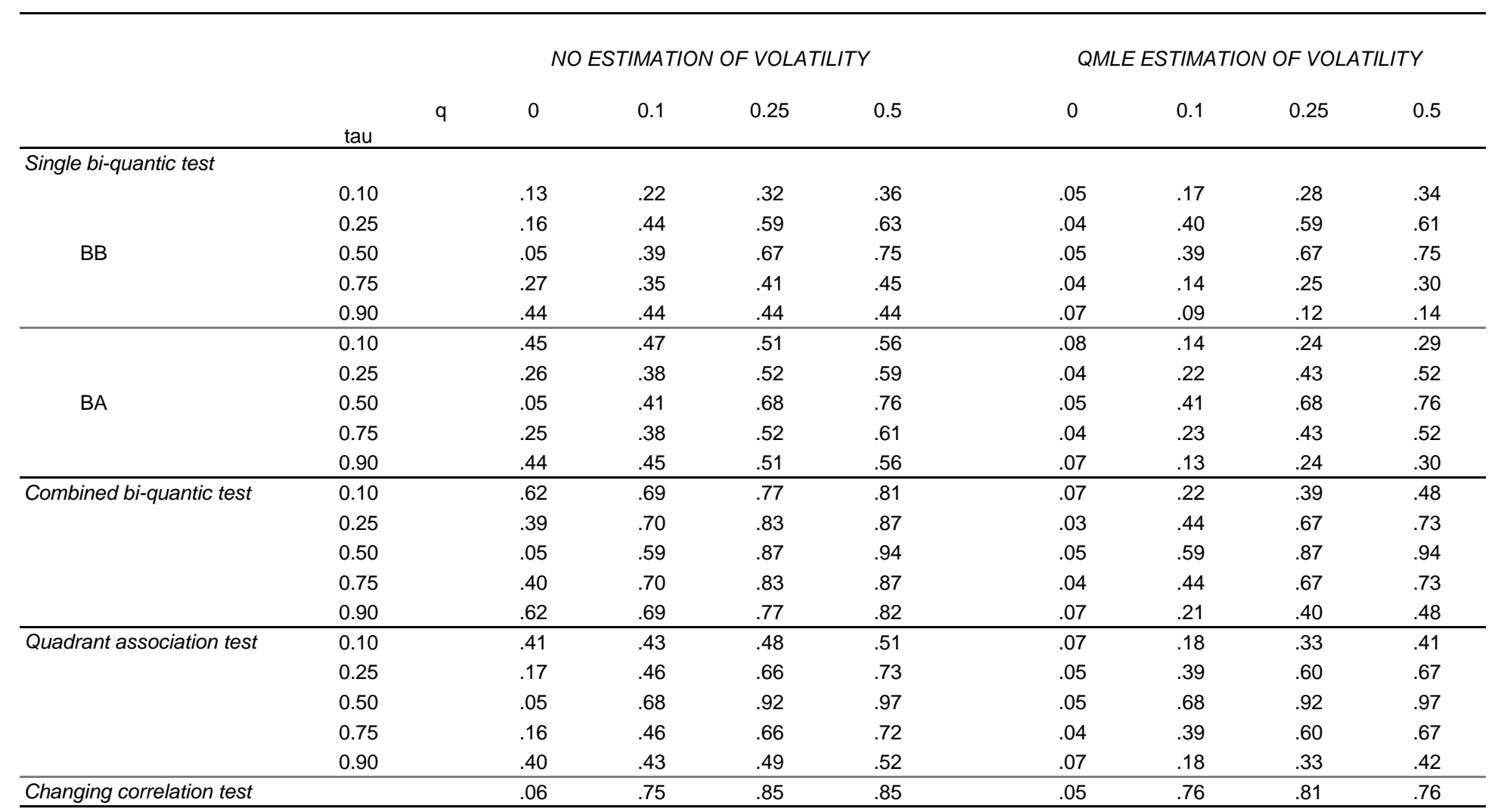


Table 4b: Structural break in correlation, with stochastic volatility. Simulated rejection frequencies of the tests: $T=300$

\begin{tabular}{|c|c|c|c|c|c|c|c|c|c|c|}
\hline & \multirow[b]{2}{*}{ tau } & \multicolumn{5}{|c|}{ NO ESTIMATION OF VOLATILITY } & \multicolumn{4}{|c|}{ QMLE ESTIMATION OF VOLATILITY } \\
\hline & & rho & $(0.75,0.75)$ & $(0.75,0.50)$ & $(0.75,0.25)$ & $(0.75,0)$ & $(0.75,0.75)$ & $(0.75,0.50)$ & $(0.75,0.25)$ & $(0.75,0)$ \\
\hline \multicolumn{11}{|l|}{ Single bi-quantic test } \\
\hline & 0.10 & & .11 & .16 & .28 & .45 & .04 & .10 & .24 & .45 \\
\hline & 0.25 & & .09 & .18 & .39 & .66 & .03 & .12 & .37 & 69 \\
\hline \multirow[t]{5}{*}{ BB } & 0.50 & & .05 & .13 & .34 & 62 & .05 & .13 & .34 & 62 \\
\hline & 0.75 & & .10 & .15 & .25 & .37 & .04 & .09 & .19 & .33 \\
\hline & 0.90 & & .16 & .18 & .22 & .26 & .04 & .06 & .10 & .13 \\
\hline & 0.10 & & .30 & .33 & .37 & .40 & .08 & .12 & .19 & .25 \\
\hline & 0.25 & & .17 & .26 & .42 & .57 & .05 & .15 & .37 & .58 \\
\hline \multirow[t]{3}{*}{ BA } & 0.50 & & .05 & .21 & .52 & .78 & .05 & .21 & .52 & .78 \\
\hline & 0.75 & & .11 & .20 & .38 & .57 & .04 & .15 & .36 & .59 \\
\hline & 0.90 & & .11 & .13 & .19 & .24 & .06 & .10 & .16 & .23 \\
\hline \multirow[t]{5}{*}{ Combined bi-quantic test } & 0.10 & & .29 & .35 & .51 & .67 & .07 & .14 & .31 & .53 \\
\hline & 0.25 & & .19 & .35 & .70 & .93 & .04 & .20 & .61 & .90 \\
\hline & 0.50 & & .05 & .29 & .77 & .98 & .05 & .29 & .77 & .97 \\
\hline & 0.75 & & .18 & .34 & .70 & .93 & .04 & .20 & .60 & .90 \\
\hline & 0.90 & & .29 & .35 & .50 & .68 & .07 & .14 & .32 & .54 \\
\hline \multirow[t]{5}{*}{ Quadrant association test } & 0.10 & & .08 & .19 & .33 & .45 & .05 & .14 & .30 & .43 \\
\hline & 0.25 & & .05 & .30 & 69 & .91 & .05 & .30 & .70 & .92 \\
\hline & 0.50 & & .05 & .43 & .89 & .99 & .05 & .43 & .89 & .99 \\
\hline & 0.75 & & .06 & .30 & .69 & .91 & .05 & .30 & .71 & .92 \\
\hline & 0.90 & & .08 & .19 & .33 & .45 & .05 & .14 & .30 & .44 \\
\hline Changing correlation test & & & .26 & .44 & .86 & 1.00 & .02 & .35 & .95 & 1.00 \\
\hline
\end{tabular}


Table 4c: Clayton copula with structural break and stochastic volatility (gaussian marginals). Simulated rejection frequencies of the tests: $T=200$

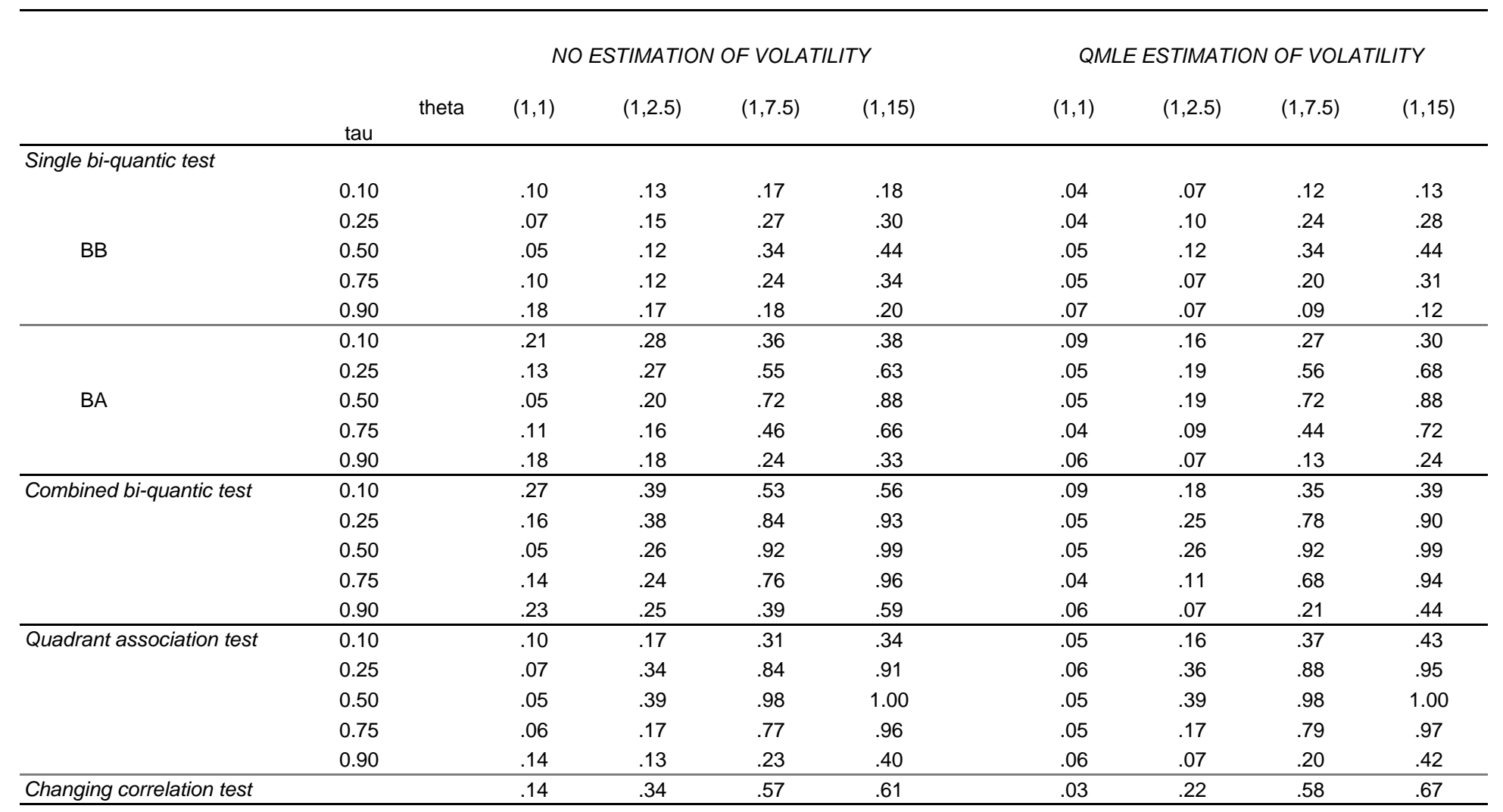

